\title{
Prevalence of esophageal eosinophilia in patients referred for diagnostic upper gastrointestinal endoscopy
}

\author{
Nancy Abdel Fattah Ahmed ${ }^{1 *}$, Hebat-Allah Moheb Amer ${ }^{2}$, Dina Abdallah Ibrahim ${ }^{3}$ and \\ Islam Abd El-Hamid El-Zayyadi²
}

\begin{abstract}
Background: Several conditions are associated with esophageal eosinophilia such as eosinophilic esophagitis (EOE) and gastro-esophageal reflux disease (GERD). The aim of this study was to detect the prevalence of esophageal eosinophilia in patients with upper gastrointestinal (Gl) symptoms referred for diagnostic upper $\mathrm{Gl}$ endoscopy. This study included 86 patients who underwent upper Gl endoscopy and biopsies.

Results: Esophageal eosinophilia EE was found in 26 patients (30.2\%): 3 patients (3.5\%) had EoE and 23 patients (26.7\%) had low-grade esophageal eosinophilia. The most common presenting symptoms were heart burn in 84 patients (97.7\%) and upper abdominal pain in 78 patients (90.7\%). Reflux esophagitis (ERD) was observed in 18.6\% of patients. In histopathological examination, EoE was found in 3.5\%, mild reflux esophagitis in $37.2 \%$, and severe reflux esophagitis in $16.3 \%$. There is statistically significant correlation between EE and male sex, hypertension, dysphagia, hiatus hernia, incompetent cardia, and fixed rings. Age, incompetent cardia, and dysphagia were statistically significant independent predictors of low-grade EE.
\end{abstract}

Conclusion: Esophageal eosinophilia EE was found in 30.2\% of patients: $3.5 \%$ had eosinophilic esophagitis EoE and 26.7\% had low-grade esophageal eosinophilia.

Keywords: EE, EoE, GERD

\section{Background}

Under physiological conditions, eosinophils are present throughout the gastrointestinal tract distal to the squamous esophagus [1]. Several conditions are associated with esophageal eosinophilia (EE) such as eosinophilic esophagitis (EoE), gastro-esophageal reflux disease (GERD), eosinophilic gastritis, gastroenteritis, or colitis with esophageal involvement, esophageal motility disorders such as achalasia, Crohn's disease with esophageal involvement, infections (fungal, viral), and hypereosinophilic syndrome $[2,3]$. In the clinical setting, some of

\footnotetext{
* Correspondence: ziad.emad90@yahoo.com

'Mansoura Specialized Medical Hospital, Internal Medicine Department, Hepatology \& Gastroenterology Unit, Faculty of Medicine, Mansoura University, Mansoura City, Egypt

Full list of author information is available at the end of the article
}

them are frequent such as GERD and EoE [2]. Eosinophilic esophagitis was first reported as the esophageal involvement of eosinophilic gastroenteritis (EGE) in 1977. Thereafter, this condition had been considered a subtype of (GERD). In 1993, Attwood et al. published the first case series of EoE as a distinct disease entity different to GERD or secondary EE [4]. Several risk factors and mechanisms have been described by which external environmental agents and factors inherent to each person may lead to EoE. The diagnosis of EoE requires all of the following: symptoms related to esophageal dysfunction, eosinophil-predominant inflammation on esophageal biopsy, characteristically consisting of a peak value of $\geq 15$ eosinophils per high-power field (HPF) (or 60 eosinophils per $\mathrm{mm}^{2}$ ), and exclusion of other causes that may be responsible for or contributing to symptoms and 
esophageal eosinophilia [2]. The most common symptom of EoE in adults is dysphagia [5]. Endoscopic findings of EoE are frequently described using the EoE endoscopic reference score (EREFS), which stands for the five key findings (edema, rings, exudates, furrows, and strictures) [6]. In addition, the endoscopic appearance of the esophagus may be normal in 10 to $25 \%$ of patients with EoE [7]. A histological diagnosis is confirmed when there are $\geq 15$ eosinophils per high-power field (HPF) [2]. Currently, the recommendation is to take at least six biopsies from two different sites, typically from the distal and proximal esophagus [8]. The diagnostic sensitivity increased to almost $100 \%$ with five or more biopsies [4]. The therapeutic approach consists of the "3D" concept: diet, drugs, and dilation [4]. Current United European Gastroenterology guidelines recommend swallowed topical corticosteroids (STCs), highdose PPI, or elimination diet for the initial treatment of EoE [9]. GERD has been defined by the Montreal Classification as a condition that occurs due to retrograde flow of gastric contents into the esophagus that lead to troublesome symptoms, which are typically heartburn and regurgitation [10]. GERD can be classified as erosive reflux disease (ERD) or non-erosive reflux disease (NERD) [11].

\section{Objectives}

To detect the prevalence of esophageal eosinophilia in patients with upper GI symptoms referred for diagnostic upper GI endoscopy.

\section{Methods}

\section{Study design}

The present study was a cross-sectional prevalence study.

\section{Settings}

The study was conducted at Mansoura Specialized Medical Hospital.

\section{Participants}

This study included 86 adult patients with unexplained upper GI symptoms referred for diagnostic upper GI endoscopy at Mansoura Specialized Medical Hospital.

\section{Variables}

\section{Inclusion criteria}

Adult patients (> 18 years) with unexplained upper GI symptoms (dysphagia, heartburn, nausea, vomiting, and upper abdominal pain) referred for diagnostic upper GI endoscopy at Specialized Medical Hospital, Mansoura University.

\section{Exclusion criteria}

Patients with the following:

- Advanced heart failure.

- Chronic liver disease.

- Chronic kidney disease.

\section{Malignancy.}

- Other causes of esophageal eosinophilia as achalasia, Crohn's disease and infections such as candidal esophagitis, connective tissue disorders, and hypereosinophilic syndrome.

\section{Data sources/measurement}

All selected patients were subjected to careful history taking, physical examination, laboratory tests including complete blood count (CBC), differential leucocytic count, serum creatinine, liver biochemical tests, abdominal ultrasound, upper GI endoscopy (esophagitis endoscopic reference score was used to minimize observer variability), biopsy (6 esophageal and 4 antral biopsies) and lastly, histopathological examination by staining the biopsies with hematoxylin and eosin (H\&E). On the high-power field, the pathologist counted the eosinophils. The presence of more than 15 eosinophils/HPF was diagnostic for EoE and presence of less than 15 eosinophils/HPF indicates low-grade EE.

\section{Bias}

N/A

\section{Study size}

The study size is determined by the statistician.

\section{Quantitative variables}

N/A

\section{Statistical methods}

Data were entered and analyzed using IBM-SPSS software (IBM Corp. Released 2017. IBM SPSS Statistics for Windows, Version 25.0. Armonk, NY: IBM Corp.)

\section{Results}

EE was found in 26 patients (30.2\%): 3 patients (3.5\%) had EoE and 23 patients (26.7\%) had low-grade esophageal eosinophilia as shown in Table 1.

The most common presenting symptoms were heart burn in 84 patients (97.7\%) and upper abdominal pain in 78 patients $(90.7 \%)$ as shown in Table 1.

Reflux esophagitis (ERD) was observed in $18.6 \%$ of patients as shown in Table 1. 
Table 1 Descriptive statistics of demographic, clinical, endoscopic and histopathological parameters of the studied cases $(N=86)$

Variable
Residence:
rural
urban
Heart burn
Upper abdominal pain
Nausea
Vomiting
Dysphagia
Unintentional weight loss
Current smoking
Atopy
DM
Hypertension
NSAIDs use
PPI use
PPI duration (months) median (min.-max.)
Reflux oesophagitis
Grade A
Grade B
Grade C
Incompetent cardia
Hiatus hernia
Fixed rings (Trachealization)
Gastritis
Antral gastritis
Pan gastritis
Histologically Oesophagitis
Mere

Eosinophilic oesophagitis (eosinophils $\geq 15 / \mathrm{HPF}$ )

Basal cell hyperplasia

$\begin{array}{lll}<\mathbf{1 5} \% & 48 & 55.8 \\ \mathbf{1 5 - 3 0 \%} & 34 & 39.5 \\ >\mathbf{3 0} \% & 4 & 4.7\end{array}$

Papillary elongation

$$
<50 \%
$$$$
50-75 \%
$$

Dilated intercellular space

Absent
$<$ one lymphocyte diameter
$\geq$ one lymphocyte diameter
Itraepithelial eosinophils

Table 1 Descriptive statistics of demographic, clinical, endoscopic and histopathological parameters of the studied cases $(N=86)$ (Continued)

\begin{tabular}{lll}
\hline Absent & 60 & 69.8 \\
$\mathbf{1 - 2}$ cells & 20 & 23.3 \\
$\mathbf{2} \mathbf{2}$ cells & 6 & 7 \\
Intraepithelial neutrophils & & \\
$\quad$ Absent & 82 & 95.3 \\
$\mathbf{1 - 2}$ cells & 2 & 2.3 \\
$\mathbf{2}$ cells & 2 & 2.3 \\
Intraepithelial mononuclear cells & & \\
$\quad \mathbf{0 - 9}$ cells & 86 & 100 \\
Chronic gastritis & 70 & 81.4 \\
H. pylori-associated gastritis & 42 & 48.8
\end{tabular}

Descriptive statistics of clinico-demographic, laboratory, endoscopic and histopathological parameters the three eosinophilic oesophagitis cases (EoE) $(N=3)$

Characteristic

Sex

Age range

Current smoking

DM, hypertension and atopy

NSAIDs use

PPI use (Duration range in months)

Dysphagia

Upper abdominal pain and heart burn

Nausea and vomiting

Unintentional weight loss

WBC count range

Eosinophil count range

Eosinophilia

Hemoglobin level range

Platelet count range

Hiatus hernia and incompetent cardia

Fixed rings

Reflux oesophagitis (ERD)

Basal cell hyperplasia

Papillary elongation

Dilated intercellular space

Intra epithelial eosinophils

Intra epithelial neutrophils

Intra epithelial mononuclear cells

\section{Statistic}

All were male patients

34-73 years

1 (33.3\%)

None

None

All (2-4 months)

All

All

None

None

5.8-6.3

0.05-0.1

Mild eosinophilia in 1 case

13.3-14.0

172-285

1 (33.3\%)

$2(66.7 \%)$

None

Present (15-30\% of total thickness)

Normal $(<50 \%)$ in all

Dilated $(<1$ lymphocyte) in all

$\geq 15 /$ HPF in all

$>2$ cells in 1 patient

No cells in the two other patients

Not present 
Table 1 Descriptive statistics of demographic, clinical, endoscopic and histopathological parameters of the studied cases $(N=86)$ (Continued)

\begin{tabular}{ll}
\hline reflux oesophagitis (histologically) & $\begin{array}{l}\text { Severe reflux } \\
\text { esophagitis in 1 } \\
\text { patient. } \\
\text { No reflux in 2 } \\
\text { other patients }\end{array}$ \\
H. pylori-associated chronic gastritis & All \\
\hline
\end{tabular}

In histopathological examination, EoE was found in $3.5 \%$, mild reflux esophagitis in $37.2 \%$, and severe reflux esophagitis in $16.3 \%$ as shown in Tables 1 and 1.

There is statistically significant correlation between EE and proportions of male sex, hypertension, dysphagia, hiatus hernia, incompetent cardia, and fixed rings as shown in Table 2.

Age, incompetent cardia, and dysphagia were statistically significant independent predictors of low-grade EE as shown in Table 3.

This table showed that 62 patients $(72.1 \%)$ live in rural areas and 24 patients (27.9\%) in urban areas. The most common symptoms were heart burn in 84 patients $(97.7 \%)$ and upper abdominal pain in 78 patients $(90.7 \%)$, while dysphagia was observed in 22 patients (25.6\%). Current smoking in 14 (16.3\%) patients, atopy in 8 (9.3\%), and PPI use in $76(88.4 \%)$ patients with median duration of 3 months (0-12).

On endoscopic examination, reflux esophagitis was observed in $18.6 \%$, incompetent cardia in $48.8 \%$ and fixed rings in $7 \%$ of patients. Gastritis was observed in 59 patients $68.6 \%$ (45 antral and 14 pan gastritis).

On histopathological examination, EE was found in 26 patients (30.2\%): 3 patients (3.5\%) had EoE and 23 patients $(26.7 \%)$ had low-grade esophageal eosinophilia (< 15 eosinophils/HPF). Mild reflux esophagitis was found in $37.2 \%$, severe reflux esophagitis in $16.3 \%$, and $\mathrm{H}$. pylori-associated gastritis in $48.8 \%$ of cases.

This table shows that EoE cases were males, and their age range from 34 to 73 years. One patient was a smoker. All 3 patients had recent history of PPI use (for 2-4 months). All patients had dysphagia, upper abdominal pain, and heart burn. No one had atopy. No one had peripheral eosinophilia. WBCs, hemoglobin, and platelets were within normal range. In upper GI endoscopy, 2 patients had fixed rings while 1 patient had normal appearing mucosa. Incompetent cardia and hiatus hernia were observed in 1 patient. ERD was not observed in any patient. According to histopathological findings, basal cell hyperplasia and dilated intercellular space were observed in all patients, but there was no papillary elongation. Intra epithelial eosinophils were $\geq 15$.
Table 2 Correlation between EE as an ordinal variable and dichotomous study variables

\begin{tabular}{lll}
\hline $\begin{array}{l}\text { Study variable } \\
\text { Rank biserial correlation } \\
\text { (dichotomous variables) }\end{array}$ & Correlation coefficient & $\boldsymbol{P}$ value \\
\hline Marital status & 0.025 & \\
Sex & -0.246 & 0.817 \\
Residence & 0.185 & $\mathbf{0 . 0 2 2}$ \\
Diabetes mellitus & 0.086 & 0.088 \\
Hypertension & 0.217 & 0.431 \\
Atopy & 0.125 & $\mathbf{0 . 0 4 5}$ \\
NSAIDs use & 0.125 & 0.252 \\
PPI use & -0.066 & 0.252 \\
Dysphagia & 0.336 & 0.548 \\
Nausea & -0.056 & $\mathbf{0 . 0 0 2}$ \\
Vomiting & 0.026 & 0.607 \\
Heartburn & 0.101 & 0.812 \\
Abdominal pain & 0.042 & 0.355 \\
Chronic gastritis & -0.186 & 0.699 \\
H. pylori & -0.011 & 0.086 \\
Hiatus hernia & 0.485 & 0.923 \\
Incompetent cardia & -0.348 & $<\mathbf{0 . 0 0 1}$ \\
Fixed rings & 0.452 & $\mathbf{0 . 0 0 1}$ \\
Weight loss & -0.084 & $<\mathbf{0 . 0 0 1}$ \\
\hline & & 0.429 \\
\hline
\end{tabular}

Table 3 Predictors of the likelihood of low-grade EE vs absent EE

\begin{tabular}{|c|c|c|c|c|c|c|}
\hline \multirow[t]{2}{*}{ Predictor } & \multicolumn{3}{|c|}{ Univariate } & \multicolumn{3}{|c|}{ Multivariate } \\
\hline & $P$ value & COR & $95 \% \mathrm{Cl}$ & $P$ value & OR & $95 \% \mathrm{Cl}$ \\
\hline Age (years) & 0.002 & & & 0.019 & & \\
\hline$<46$ years & & R & R & & R & R \\
\hline$\geq 46$ years & & 5.2 & $1.8-14.7$ & & 4.4 & $1.3-15$ \\
\hline Cardia & 0.001 & & & 0.007 & & \\
\hline Competent & & R & $\mathrm{R}$ & & $\mathrm{R}$ & R \\
\hline Incompetent & & 8.2 & $2.5-27.2$ & & 5.8 & $1.6-20.9$ \\
\hline Dysphagia & 0.034 & & & 0.033 & & \\
\hline Absent & & R & R & & R & R \\
\hline Present & & 3.2 & $1.1-9.4$ & & 4.3 & $1.1-16.6$ \\
\hline Hypertension & 0.023 & & & 0.203 & & \\
\hline Absent & & $\mathrm{R}$ & R & & $R$ & R \\
\hline Present & & 4.9 & $1.2-19.6$ & & 2.7 & $0.583-12.6$ \\
\hline
\end{tabular}

$R=$ reference category, $C O R=$ crude odds ratio, $O R=$ odds ratio, $C l=$ confidence interval, $P$ value binary logistic regression 
There was severe reflux esophagitis in the patient. All 3 patients had $\mathrm{H}$. pylori in gastric biopsy.

This table showed a statistically significant correlation between EE and proportions of male sex, hypertension, dysphagia, hiatus hernia, incompetent cardia, and fixed rings.

As the proportions of male sex, hypertension, dysphagia, hiatus hernia, incompetent cardia, and fixed rings go up, the grading of EE goes up. No statistically significant correlation with other dichotomous variables.

This table showed the results of binary logistic regression analysis that was run to ascertain the effects of age $\geq 46$ years, incompetent cardia, presence of dysphagia, and hypertension on the likelihood that participants will exhibit low-grade EE. Univariate analysis showed that each of the four predictor variables was statistically significant. On running multivariate analysis, only age, incompetent cardia, and dysphagia were statistically significant independent predictors of low-grade EE.

The model was statistically significant $\left(\chi^{2}[4]=27.338\right.$, $\underline{P}<0.001)$.

The model correctly classified $75.9 \%$ of cases with sensitivity of $56.5 \%$ and specificity of $83.3 \%$.

Participants with older age ( $\geq 46)$, incompetent cardia, and dysphagia had 4.4, 5.8, and 4.3 times higher odds to exhibit low-grade EE.

\section{Participants}

This study included 86 adult patients with unexplained upper GI symptoms referred for diagnostic upper GI endoscopy at Mansoura Specialized Medical Hospital.

\section{Descriptive data, outcome data, and main results}

Descriptive data, outcome data, and main results are shown in Tables 1, 2 and 3.

\section{Other analyses}

N/A

\section{Key results}

EE was found in 26 patients (30.2\%): 3 patients (3.5\%) had EoE and 23 patients (26.7\%) had low-grade esophageal eosinophilia.

The most common presenting symptoms were heart burn in 84 patients (97.7\%) and upper abdominal pain in 78 patients $(90.7 \%)$. Reflux esophagitis (ERD) was observed in 16 patients (18.6\%). In histopathological examination, EoE was found in 3 patients (3.5\%), mild reflux esophagitis in 32 patients (37.2\%), and severe reflux esophagitis in 14 patients (16.3\%). EoE patients were 3 males and had dysphagia but no history of atopy. Two EoE patients had fixed rings while 1 patient had normal appearing mucosa. There is statistically significant correlation between EE and proportions of male sex, hypertension, dysphagia, hiatus hernia, incompetent cardia, and fixed rings. Age, incompetent cardia, and dysphagia were statistically significant independent predictors of low-grade EE.

\section{Discussion}

The present study included 86 patients with upper GI symptoms referred for upper GI endoscopy. Eosinophils are not present in the esophagus under normal conditions [1]. Eosinophilic esophagitis diagnosis should be confirmed histologically based on the presence of more than 15 eosinophils per high-power field and the exclusion of other causes of eosinophilia [7].

Therefore, the aim of our study was to detect the prevalence of esophageal eosinophilia in patients with upper gastrointestinal (GIT) symptoms referred for diagnostic upper GIT endoscopy. In the current study, 14 patients $(16.3 \%)$ were smokers, 4 patients $(4.7 \%)$ had D.M, 10 patients $(11.6 \%)$ had hypertension, 8 patients $(9.3 \%)$ had atopy, 8 patients $(9.3 \%)$ had history of recent NSAIDs use, and 76 patients $(88.4 \%)$ were with recent history of PPIs use with median duration of 3 months. In similar study conducted by Hunter et al. (2014) [12] on 91 adult patients presenting with various upper gastrointestinal symptoms, they found that $61.5 \%$ of patients were males and $38.4 \%$ were females. One third of the patients gave history of smoking and $71 \%$ gave history of PPIs use. In our study, the most common presenting symptom was heart burn which is present in 84 patients (97.7\%) followed by upper abdominal pain in 78 patients (90.7\%). In contrast, in an Egyptian study conducted in El-Minia University, the upper abdominal pain was the most common symptom and reported in $63.3 \%$ then heartburn in $50 \%$ of patients [13]. In our study, the most common histopathological finding was that of reflux esophagitis which was found in 46 cases (53.5\%): mild reflux esophagitis in 32 cases (37.2\%) and severe reflux esophagitis in 14 cases (16.3\%). EoE was observed in 3 cases (3.5\%); 1 case of them has histopathological findings of reflux esophagitis. Thirty-eight cases had normal esophageal biopsy. H. pylori was found in 42 cases $(48.8 \%)$ of cases.

In our study, we found 26 cases (30.2\%) with EE, 3 cases $(3.5 \%)$ had high-grade esophageal eosinophilia ( $\geq$ $15 / \mathrm{HPF}$ ) which fulfill the diagnostic criteria of eosinophilic esophagitis, 23 cases (26.7\%) had low-grade esophageal eosinophilia $(<15 / \mathrm{HPF})$, but 60 cases $(69.8 \%)$ had absent esophageal eosinophilia.

Also, there is an Egyptian study that showed the prevalence of EoE in patients with upper GI symptoms was $1.8 \%$ [13].

This discrepancy in prevalence rate is related to many factors: first, differences in demographic data 
(i.e., age group, gender predominance, race, and ethnicity) of patients included in each study; second, using different diagnostic criteria of eosinophilic esophagitis; and third, the complex interplay between eosinophilic esophagitis and GERD which can lead to many conflicting results [14].

In our study, the 3 cases of eosinophilic esophagitis were male, and their age range was from 34 to 73 years. One case was a smoker. All 3 cases had recent history of PPI use (for 2-4 months).

All 3 patients had dysphagia, upper abdominal pain, and heart burn. None had atopy nor peripheral eosinophilia.

Sawada et al. (2019) [15] analyzed a total of 106 patients with EoE and a median age of 46 years (range 4152). Similarly, the majority of patients were male $(65 \%)$ and most patients $(89 \%)$ had symptoms including dysphagia $(69 \%)$. However, heartburn and chest pain represented (25 and 15\%), respectively, and $70 \%$ of patients had comorbidities of allergic diseases.

In agreement with our study, Fouad et al. (2018) [13] had found that EoE is more common in males than in females (3:1) with mean age $34.3 \pm 6.0$ years. But no one was smoker and all had normal stomach and duodenal endoscopic findings.

In the present study, reflux esophagitis (ERD) was observed in $18 \%$ by endoscopy while by biopsy, we found mild reflux esophagitis in $37.2 \%$ and severe reflux esophagitis in $16.3 \%$ in spite of normal appearing mucosa.

So, it is suggested to obtain an endoscopic biopsy in spite of normal appearing lower esophageal mucosa in patients with GERD symptoms, in those with incompetent cardia, in those who are currently smoking, or in those with hiatus hernia, particularly in male patients.

In our study, we found statistically significant correlation between EE and proportions of male sex, hypertension, dysphagia, hiatus hernia, incompetent cardia, and fixed rings.

Also age, incompetent cardia, and dysphagia were statistically significant independent predictors of low-grade EE.

Similarly, Ravi et al. 2011 [16] found that patients with low-grade esophageal eosinophilia were older $(48 \pm 2.1)$ and showed a male predominance. Dysphagia was the most common presenting symptom as present in $83 \%$ of patients, and the fixed esophageal rings was the most common endoscopic finding, as seen in 37\% of patients.

In the current study, the fixed rings (trachealization) were present in 6 patients (7\%): 2 of them were diagnosed to have EoE and the other 4 patients had low-grade EE. This can be explained by that the fixed rings are nonpathognomonic as mentioned by Braunberger et al. (2020) [17]. Our 3 EoE cases had recent history of PPI use (for 2-
4 months) with no improvement of their symptoms which exclude PPI-responsive esophageal eosinophilia (PPI-REE) as there are no significant clinical, histological, and endoscopic characteristics that distinguish PPI-REE from EoE (Eluri and Dellon 2015) [18] despite the efficacy of PPI as stated by Young, 2020 [19].

\section{Limitations}

Considering the limited subjects, only 86 patients were included in the study, so our results should be interpreted with caution. Further studies are needed to evaluate patients with low-grade eosinophilia.

\section{Interpretation}

Our results should be interpreted with caution because of several limitations. We recruited 86 patients in this study, and the sample size is relatively small which may restrict the subgroups analysis. All participants were from Mansoura Specialized Medical Hospital which may not stand for all the Egyptian population.

\section{Generalizability}

The fundamental experiments should be further conducted to validate our results and explore the possible mechanism.

\section{Conclusion}

EE was found in 26 patients (30.2\%): 3 patients (3.5\%) had EoE and 23 patients (26.7\%) had low-grade EE. EoE prevalence has evolved in the last 2 decades and varies according to different population. Many predictors for low-grade EE has been identified but still its clinical significance is not clearly defined and further studies are recommended.

\section{Abbreviations}

EE: Esophageal eosinophilia; EoE: Eosinophilic esophagitis; GERD: Gastroesophageal reflux disease; ERD: Erosive reflux disease; NERD: Non-erosive reflux disease; GI: Gastrointestinal; HPF: High-power field; H\&E: Hematoxylin and eosin; DM: Diabetes mellitus; PPI: Proton pump inhibitor; PPI-REE: PPIresponsive esophageal eosinophilia; EGE: Eosinophilic gastroenteritis

\section{Acknowledgements}

Thanks to every person who shared in this work.

\section{Authors' contributions}

NAFA: manuscript review, editing, design, and publishing. HAMA: literature search, clinical follow-up, and statistics. DAAI: histopathological studies. IAEE: idea of the study and data collection. All authors have read and approved the manuscript.

\section{Funding}

Not applicable.

Availability of data and materials

The datasets used and/or analyzed during the current study are available from the corresponding author on reasonable request. 


\section{Declarations}

\section{Ethics approval and consent to participate}

The study protocol was investigated and approved by the Medical Ethics Research Team, Faculty of Medicine, Mansoura University (code number: MS.18. 12.392). Every case, after guaranteeing privacy, has been given verbal consent as most of the patients cannot read or write.

\section{Consent for publication}

Not applicable.

\section{Competing interests}

The authors declare that they have no competing interests.

\section{Declarations}

\section{Ethics approval and consent to participate}

The study protocol was investigated and approved by the Medical Ethics Research Team, Faculty of Medicine, Mansoura University (code number: MS.18. 12.392). Every case, after guaranteeing privacy, has been given verbal consent as most of the patients cannot read or write.

\section{Consent for publication}

Not applicable.

\section{Competing interests}

The authors declare that they have no competing interests.

\section{Author details}

'Mansoura Specialized Medical Hospital, Internal Medicine Department, Hepatology \& Gastroenterology Unit, Faculty of Medicine, Mansoura University, Mansoura City, Egypt. ${ }^{2}$ Specialized Medical Hospital, Internal Medicine Department, Hepatology \& Gastroenterology Unit, Faculty of Medicine, Mansoura University, Mansoura City, Egypt. ${ }^{3}$ Pathology

Department, Faculty of Medicine, Mansoura University, Mansoura City, Egypt.

Received: 22 March 2021 Accepted: 13 June 2021

Published online: 30 July 2021

\section{References}

1. Walker MM, Potter M, Talley NJ (2018) Eosinophilic gastroenteritis and other eosinophilic gut diseases distal to the oesophagus. Lancet Gastroenterol Hepatol 3(4):271-280. https://doi.org/10.1016/S2468-1253(18)30005-0

2. Dellon ES et al (2018) Updated international consensus diagnostic criteria for eosinophilic esophagitis: proceedings of the AGREE conference. Gastroenterology 155(4):1022-1033.e10

3. Reed CC, Dellon ES (2019) Eosinophilic esophagitis. Med Clin North Am 103(1):29-42. https://doi.org/10.1016/j.mcna.2018.08.009

4. et al (2017) Diagnosis and treatment of eosinophilic esophagitis in clinical practice. Clin J Gastroenterol 10(2):87-102. https://doi.org/10.1007/s12328-0 7-0725-4Abe, Y.et al, Diagnosis and treatment of eosinophilic esophagitis in clinical practice. Clin J Gastroenterol, 2017. 10(2): p. 87-102, DOI: https://doi. org/10.1007/s12328-017-0725-4.

5. Mari A, Tsoukali E, Yaccob A (2020) Eosinophilic esophagitis in adults: a concise overview of an evolving disease. Korean J Fam Med 41(2):75-83. https://doi.org/10.4082/kjfm.18.0162

6. Lucendo AJ, Arias Á (2017) The role of endoscopy in eosinophilic esophagitis: from diagnosis to therapy. 11(12):1135-1149

7. Gomez-Aldana A et al (2019) Eosinophilic esophagitis: current concepts in diagnosis and treatment. World J Gastroenterol 25(32):4598-4613. https:// doi.org/10.3748/wjg.v25.132.4598

8. Gomez Torrijos E, Gonzalez-Mendiola R, Alvarado M, Avila R, Prieto-Garcia A, Valbuena T, Borja J, Infante S, Lopez MP, Marchan E, Prieto P, Moro M, Rosado A, Saiz V, Somoza ML, Uriel O, Vazquez A, Mur P, Poza-Guedes P, Bartra J (2018) Eosinophilic esophagitis: review and update. Front Med 5 247. https://doi.org/10.3389/fmed.2018.00247

9. Miehlke $\mathrm{S}$ et al (2020) Orodispersible budesonide tablets for the treatment of eosinophilic esophagitis: a review of the latest evidence. Ther Adv Gastroenterol 13:1756284820927282
10. Wong S, Ruszkiewicz A, Holloway RH, Nguyen NQ (2018) Gastrooesophageal reflux disease and eosinophilic oesophagitis: what is the relationship? World J Gastrointest Pathophysiol 9(3):63-72. https://doi.org/1 0.4291/wjgp.v9.i3.63

11. Kellerman R, Kintanar T (2017) Gastroesophageal reflux disease. Prim Care 44(4):561-573. https://doi.org/10.1016/j.pop.2017.07.001

12. Hunter S, Helmy D, Zayed N, El-Tayeb T, El-Serafy M (2014) Eosinophilic esophagitis in Egyptian adult patients presenting with upper gastrointestinal symptoms. Open J Gastroenterol 4(02):88-95. https:/doi.org/10.4236/ojgas.2014.42015

13. Fouad M, Fouad YM, Mokareb HA, Mohamed EA, Abdel-Rehim DM (2018) Prevalence of eosinophilic esophagitis in adult patients with upper gastrointestinal symptoms in a locality in upper Egypt. Clin Endosc 51(4): 357-361. https://doi.org/10.5946/ce.2017.166

14. Hirano I (2011) Eosinophilic esophagitis and gastroesophageal reflux disease: there and back again. Clin Gastroenterol Hepatol 9(2):99-101. https://doi.org/10.1016/j.cgh.2010.11.001

15. Sawada A, Hashimoto A, Uemura R et al (2019) Association between endoscopic findings of eosinophilic esophagitis and responsiveness to proton pump inhibitors. Endoscopy Int Open 7(4):E433

16. Ravi K, Talley NJ, Smyrk TC, Katzka DA, Kryzer L, Romero Y, Arora AS, Alexander JA (2011) Low grade esophageal eosinophilia in adults: an unrecognized part of the spectrum of eosinophilic esophagitis? Dig Dis Sci 56(7):1981-1986. https://doi.org/10.1007/s10620-011-1594-1

17. Braunberger, R., Hanson, J. and Gonzalez, R. (2021) Eosinophilic esophagitis. Esophagus. Pathology outlines.com.

18. Eluri S, Dellon ES (2015) Proton pump inhibitor-responsive oesophageal eosinophilia and eosinophilic oesophagitis: more similarities than differences. Curr Opin Gastroenterol 31(4):309-315. https://doi.org/10.1097/MOG.0000000000000185

19. Young A (2020) Real-world data show efficacy of PPIs in EoE. 21 July 2020. Healio news. Gastroenterology.

\section{Publisher's Note}

Springer Nature remains neutral with regard to jurisdictional claims in published maps and institutional affiliations.

\section{Submit your manuscript to a SpringerOpen ${ }^{\circ}$ journal and benefit from:}

- Convenient online submission

- Rigorous peer review

- Open access: articles freely available online

- High visibility within the field

Retaining the copyright to your article

Submit your next manuscript at $\boldsymbol{\nabla}$ springeropen.com 